\title{
Mixed Convection in the VHTR in the Event of a LOFA
}

Richard W. Johnson

May 2012

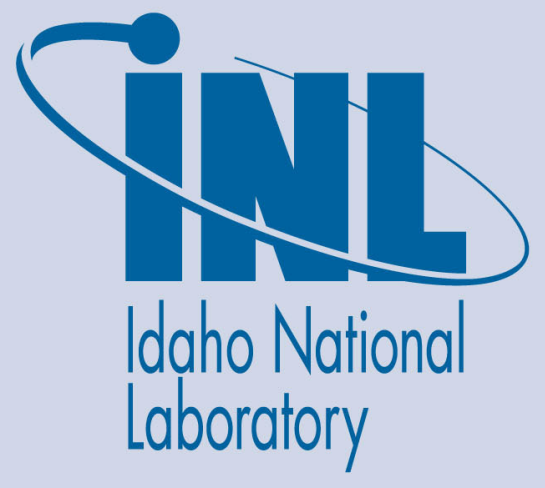

The INL is a U.S. Department of Energy National Laboratory operated by Battelle Energy Alliance 
INL/EXT-12-26039

\title{
Mixed Convection in the VHTR in the Event of a LOFA
}

\author{
Richard W. Johnson
}

May 2012

\section{Idaho National Laboratory \\ Idaho Falls, Idaho 83415}

http://www.inl.gov

Prepared for the

U.S. Department of Energy

Office of Nuclear Energy

Under DOE Idaho Operations Office

Contract DE-AC07-05ID14517 
Mixed Convection in the VHTR in the Event of a LOFA

\author{
Richard W. Johnson \\ Thermal Science and Safety Analysis \\ Idaho National Laboratory
}

\begin{abstract}
The US Department of Energy, Office of Nuclear Energy (DOE-NE) is supporting the development of a very high temperature reactor (VHTR) concept as the primary focus of it next generation nuclear power plant (NGNP) program. The VHTR is cooled by forcing helium downwards through the core into the lower plenum and out the hot duct. In the event that the coolant circulators are lost, the driving pressure drop across the core will reduce to zero and there will be the opportunity for natural circulation to occur. During the time that the circulators are powering down, the heat transfer in the core from the graphite blocks to the helium coolant will transform from turbulent forced convection to mixed convection, where buoyancy effects become important, to free or natural convection, where buoyancy is dominant. Analysis of the nature of the forced, mixed and free convection is best done using computational fluid dynamic (CFD) software that can provide fine details of the flow and heat transfer. However, CFD analysis involves approximations in the results because of the finite nature of the spatial and temporal discretizations required, the inexact nature of the turbulence models used, approximations used in the describing equations, such as the Boussinesq approximation where the density is allowed to vary only in the gravitational body force term, and the finite precision of the computers employed. Therefore, it is necessary to validate the CFD computations. Validation is accomplished by comparing results from specific CFD computations to experimental data that have been taken specifically for the purpose of validation and that are related to the physical phenomena in question. The present report examines the flow and heat transfer parameters (dimensionless numbers) that characterize the flow and reports ranges for their values based on specific CFD studies performed for the VHTR.

\section{Introduction}

The US Department of Energy, Office of Nuclear Energy (DOE-NE) is supporting the development of a very high temperature reactor (VHTR) concept as the primary focus of it next generation nuclear power plant (NGNP) program. The VHTR has two distinct design concepts that have been promoted by different reactor vendors, the pebble-bed and the prismatic concepts. In both cases, TRISO fuel, consisting of small uranium particles in the range of $1-2 \mathrm{~mm}$ each having received multiple coats of special materials are then either compacted into tennis ball sized "pebbles" for the pebble bed reactor or cylindrical pins for the prismatic design. In the prismatic design, hexagonal cross-sectioned graphite blocks are drilled to accommodate the fuel pins and provide coolant channels to remove the thermal energy. The coolant channels extend the height of the core. Figure 1 illustrates the cross-section of a prismatic VHTR core, where there are 66 stacks of fueled blocks, and a close-up of a single fueled block. The fuel pin channels are shown in the single fuel block as smaller darker circles while the coolant channels are shown as larger lighter circles.
\end{abstract}




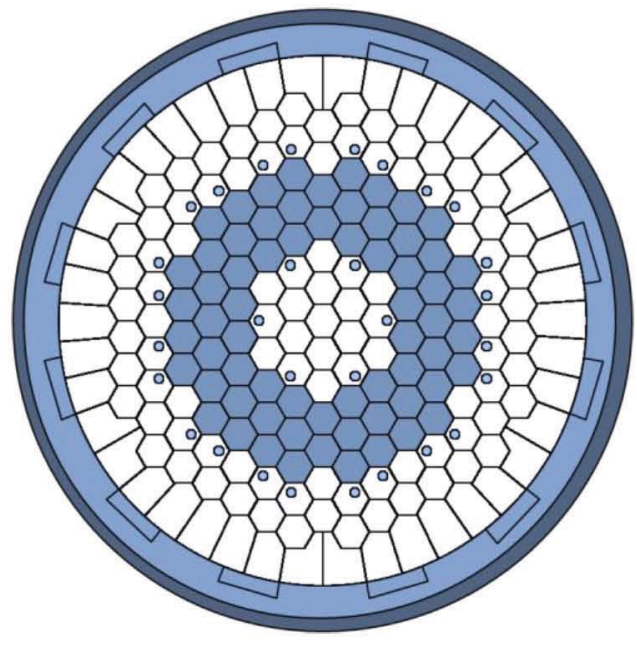

(a)

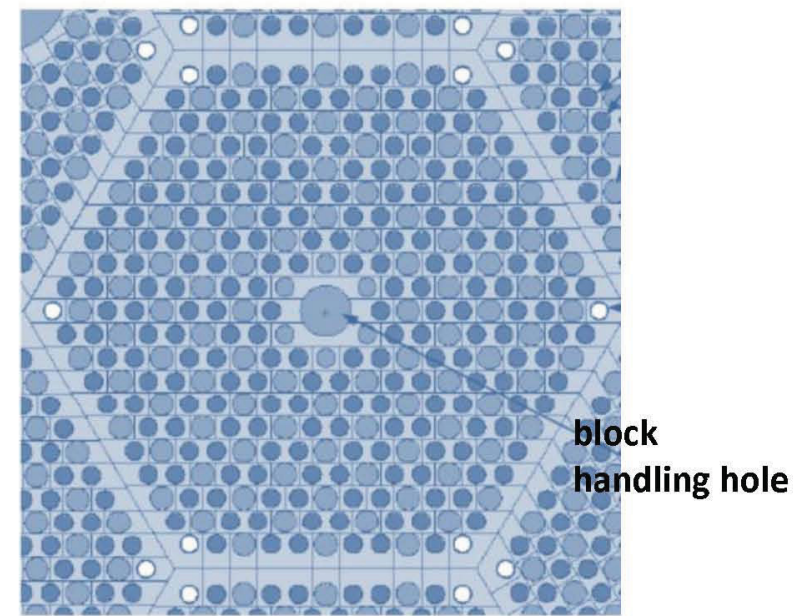

(b)

Figure 1. (a) Cross section of the VHTR core; (b) close-up of a prismatic block.

In the event that the coolant circulators are lost, the driving pressure drop across the core will reduce to zero and there will be the opportunity for natural circulation to occur. During the time that the circulators are powering down, the heat transfer in the core from the graphite blocks to the helium coolant will transform from turbulent forced convection to mixed convection, where buoyancy effects become important, to free or natural convection, where buoyancy is dominant. Analysis of the nature of the forced, mixed and free convection is best done using computational fluid dynamic (CFD) software that can provide fine details of the flow and heat transfer. However, CFD analysis involves approximations in the results because of the finite nature of the spatial and temporal discretizations required, the inexact nature of the turbulence models that are used, approximations used in the describing equations, such as the Boussinesq approximation where the density is allowed to vary only in the gravitational body force term, and the finite precision of the computers employed. Therefore, it is necessary to validate the CFD computations. Validation is accomplished by comparing results from specific CFD computations to experimental data that have been taken specifically for the purpose of validation and that are related to the physical phenomena in question. The Nuclear Energy University Program (NEUP) is designed to provide opportunities to the academic community to help provide technical support to the programs of the DOENE. The focus of the NEUP-supported project entitled "Transient Mixed Convection Validation for NGNP" is to perform experiments that can be used for CFD validation purposes for the events during which the helium circulators are lost and where there may or may not be a break in the piping system where air can then ingress into the reactor vessel (RV). Such an accident will involve transient physics as mentioned above where forced convection becomes mixed convection, which then becomes free convection. During the initial normal operating conditions and then during the accident transient, the helium coolant will experience a large range in temperature variation, from about $530 \mathrm{~K}$ at the top of the core to upwards of $1300 \mathrm{~K}$ below the core; the helium is designed to flow downwards through the core during normal operation. Such a large temperature variation will provide a strong potential for significant buoyancy effects in the flow and heat transfer. The typical engineering parameters that are most effective in characterizing the flow and heat transfer are derived from the conservation equations for momentum and energy.

\section{Describing Equations}


The conservation equation for momentum is given as follows [1]:

$$
\rho \frac{\partial u_{j}}{\partial t}+\rho u_{k} \frac{\partial u_{j}}{\partial x_{k}}=-\frac{\partial p}{\partial x_{j}}+\frac{\partial}{\partial x_{i}}\left[\mu\left(\frac{\partial u_{i}}{\partial x_{j}}+\frac{\partial u_{j}}{\partial x_{i}}\right)\right]+\rho f_{j}
$$

where $f$ is a body force such as gravity.

For relatively low-speed gas flow where there are significant changes in density because of variations in temperature, one can expand the density as follows [2]:

$$
\rho=\rho_{o}+\Delta \rho \approx \rho_{o}(1-\beta \Delta T) \quad \text { where } \beta=-\frac{1}{\rho}\left(\frac{\partial \rho}{\partial T}\right)_{p}
$$

Substituting Eq. (2) into Eq. (1) for the density present in the body force and assuming that gravity is the only important body force acting on the flow, one obtains what is called the Boussinesq approximation where the reference density is used for the other terms [2]:

$$
\rho_{o} \frac{\partial u_{j}}{\partial t}+\rho_{o} u_{k} \frac{\partial u_{j}}{\partial x_{k}} \approx-\frac{\partial p}{\partial x_{j}}+\frac{\partial}{\partial x_{i}}\left[\mu\left(\frac{\partial u_{i}}{\partial x_{j}}+\frac{\partial u_{j}}{\partial x_{i}}\right)\right]-\rho_{o} g_{j}-\rho_{o} g_{j} \beta\left(T-T_{o}\right)
$$

Upon non-dimensionalizing the momentum equation, one obtains the following [2]:

$$
\frac{\partial u_{j}^{*}}{\partial t^{*}}+u_{k}^{*} \frac{\partial u_{j}^{*}}{\partial x_{k}^{*}} \approx-\frac{\partial P^{*}}{\partial x_{j}^{*}}+\frac{1}{R e} \frac{\partial}{\partial x_{i}^{*}}\left(\frac{\partial u_{i}^{*}}{\partial x_{j}^{*}}+\frac{\partial u_{j}^{*}}{\partial x_{i}^{*}}\right)-\frac{G r}{R e^{2}} \beta^{*} T^{*} g_{j}^{*}
$$

where the $\rho_{o} g_{j}$ term has been subsumed into the pressure gradient and the asterisk indicates a dimensionless quantity. The main consequence of performing the above non-dimensionalization of the momentum equation is the appearance of two dimensionless parameters. These are the Reynolds number and the Grashof number, defined as follows:

$$
R e=\frac{\rho_{o} V_{o} L}{\mu_{o}} \quad \text { and } \quad G r=\frac{\rho_{o}^{2} \beta_{o} g L^{3}\left(T-T_{o}\right)}{\mu_{o}^{2}}
$$

where the subscript "o" refers to a reference state (temperature and pressure), except that $V_{o}$ and $L$ refer to characteristic velocity and length scales, respectively. In the cases of both the NEUP geometry and the VHTR, the duct/coolant channels are vertical and represent the primary flow paths for the flowing fluids. There are two length scales " $L$ " that could be used in the Re and Gr numbers above to characterize the flows. These are the hydraulic diameter of the coolant channel and the height of the coolant channel. In the case of the Reynolds number, the obvious choice is to use the hydraulic diameter. In deriving the dimensionless numbers, only one length scale is used to perform the nondimensionalization. It seems that this should be the hydraulic diameter, both because it is the obvious choice for the Reynolds number, and because only one length scale is called for.

The fluid properties are defined to be at the reference temperature and pressure denoted by the subscript "o". The other parameter that needs to be defined is the temperature difference; that is, what to use for $\left(T-T_{0}\right)$. In view of the fact that the temperature difference comes from the change in density, Eq. (2), and that the buoyant force is equivalent to the weight of the displaced fluid (that is the variation in the weight of the fluid that is due to a density difference) that the temperature difference should be related to 
the axial variation in the fluid temperature as it flows through the duct; that is, $\left(T-T_{0}\right) \sim\left(T_{\text {out }}-T_{\text {in }}\right)$. Hence, one can define a datum level for the reference temperature and pressure such as those in the lower plenum of the VHTR or at the inlet to the duct in the experiment. The other temperature would be at the upper plenum or at the top of the experimental duct.

The relative strengths of forced versus free convection are said to be determined by the relationship between the Reynolds and Grashof numbers. This is indicated by the last term in Eq. (4), which is modified by the factor $\mathrm{Gr} /\left(\operatorname{Re}^{2}\right)$. That is [2], for

$$
\begin{array}{ll}
\mathrm{Gr} \ll \mathrm{Re}^{2} & \text { forced convection dominates, } \\
\mathrm{Gr} \gg \mathrm{Re}^{2} & \text { free convection dominates, } \\
\mathrm{Gr} \sim \mathrm{Re}^{2} & \text { mixed free and forced convection occurs. }
\end{array}
$$

In the nondimensionalization of the energy conservation equation, it is found that for low speed flow, such as is expected in the VHTR, the only two parameters that appear in the equations are the Reynolds and Prandtl numbers. It is then obvious that the Grashof, Reynolds and Prandtl numbers for the VHTR in the case of a loss of circulator accident be estimated to help in the design of the NEUP experiments.

\section{VHTR Simulations}

A number of simulations have been performed for the case of a loss of flow accident (LOFA) in a VHTR. A symmetric one-twelfth sector of a column of graphite blocks, see Fig. 1(b), is the basic CFD model used in the study of Tung and Johnson [3]. Figure 2 illustrates a cross-sectional view of the CFD model and shows the grid used. As can be seen, there are coolant channels (two sizes), fuel channels and a gap at the left that represents the gap between blocks into which helium coolant can flow, bypassing the coolant channels. The gap flow is denoted bypass flow.

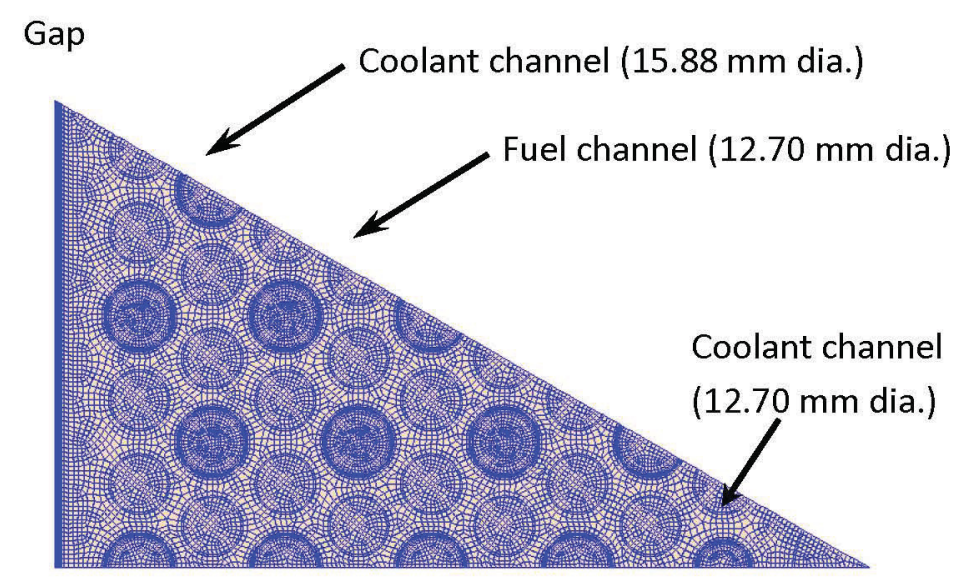

Figure 2. Cross sectional view of the CFD model with mesh.

The CFD model employs an axial "cosine" distribution of heat generation in the model. The heat generation is based on the following equation:

$$
q^{\prime \prime \prime}=A_{r} q_{\operatorname{con}}\left\{1+\left(A_{p}-1\right) \sin \left[\frac{\pi(z-1.189)}{L}\right]\right\}
$$


where $\mathrm{z}$ is the axial coordinate, $A_{r}$ is the radial factor, $A_{p}$ is the peak axial factor given as $1.3, \mathrm{~L}$ is the length of the heated section $(7.93 \mathrm{~m}), 1.189$ is thickness of the upper reflector and $q_{c o n}$ is a constant that is tuned to provide the total core heat generation and is set to $21,103,716 \mathrm{~W} / \mathrm{m}^{3}$ for the $350 \mathrm{MW}_{\text {th }}$ VHTR being considered. The peak radial factor, given as $A_{r}=1.25$, is the expected maximum factor by which the heat generation rate will exceed the average heat generation rate $\left(A_{r}=1.0\right)$ in the radial (lateral) coordinate in the core. Figure 3 plots the heat generation rate for the average and the peak radial factors.

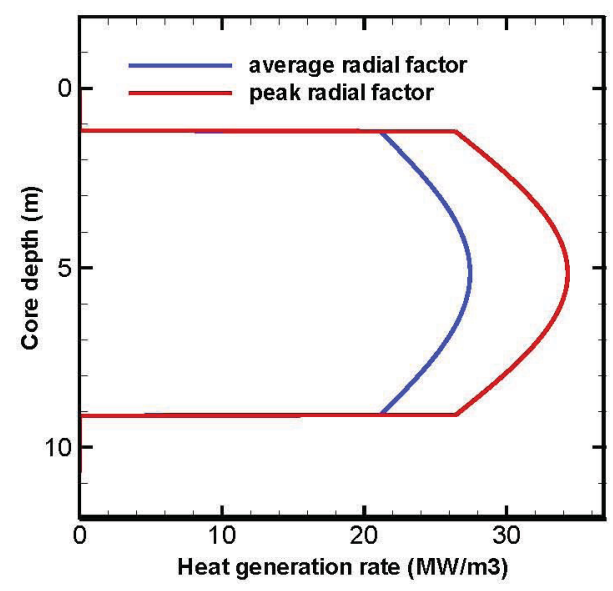

Figure 3. Average and peak heat generation.

Initial conditions for the LOFA transient are obtained by performing a steady state calculation for normal operation. For this calculation, the inlet stagnation pressure is set to $29.9 \mathrm{kPa}$ and the inlet temperature is set to $532 \mathrm{~K}\left(259^{\circ} \mathrm{C}\right)$. Graphite surfaces are assumed to be smooth. Thermal and transport properties for the helium are found from the National Institute of Standards and Technology (NIST) ${ }^{1}$; the properties are for 6.4 MPa and are assumed to be isobaric. Graphite properties are obtained from General Atomics [4]. The fuel compact properties are those used in MacDonald et al. [5]. Figure 4 illustrates the initial conditions at the hottest plane in the stack for a case of two one-twelfth sectors (where one is the mirror image of the other across the gap). The 1/12 sector on the left employs heat generation for the peak radial factor $\left(A_{r}=1.25\right)$ while that on the right is set to the average radial factor $\left(A_{r}=1.0\right)$. Note how the temperatures near the bypass gap are relatively cooler than elsewhere; this is due to the cooling effectiveness of the bypass flow in the gap. At the beginning of the LOFA transient, the pressure drop is set to zero and the heat generation rate is set to $10 \%$ of the power for normal operation. Natural circulation is allowed to eventually occur. Figure 5 shows the temperature contours at the hot plane and velocity contours at the top of the core after 100 seconds into the LOFA transient. It is obvious that the cooler region near the bypass flow gap is still cooler than the other regions. It can also be seen that natural circulation flow paths have been established between the hot and cool sectors, with the strongest up flow in the vicinity of the hottest region and the strongest down flow in the vicinity of the coolest region (near the gap).

\footnotetext{
${ }^{1}$ http://webbook.nist.gov/chemistry/fluid/
} 


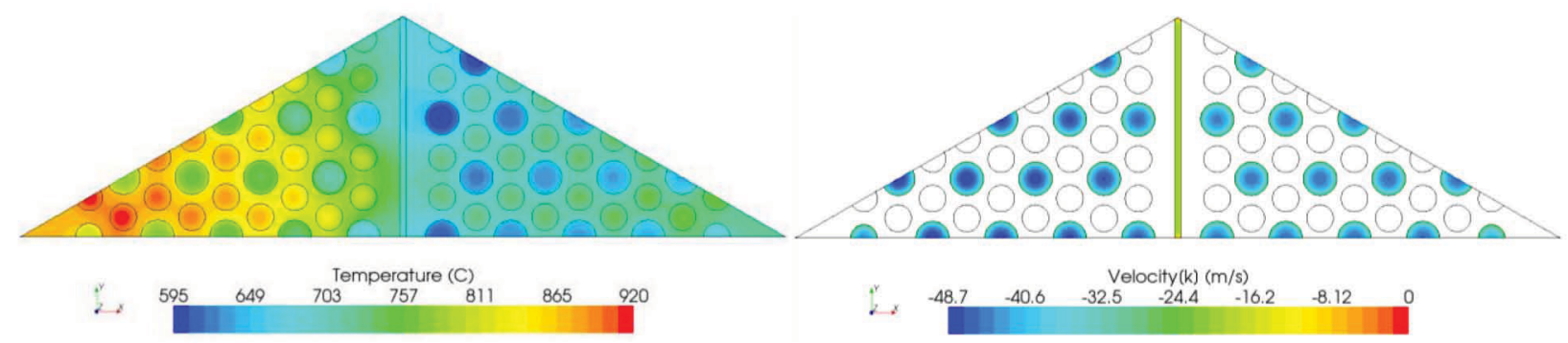

(a)

(b)

Figure 4. Contours of (a) temperature and (b) velocity at the hottest plane for normal operation.

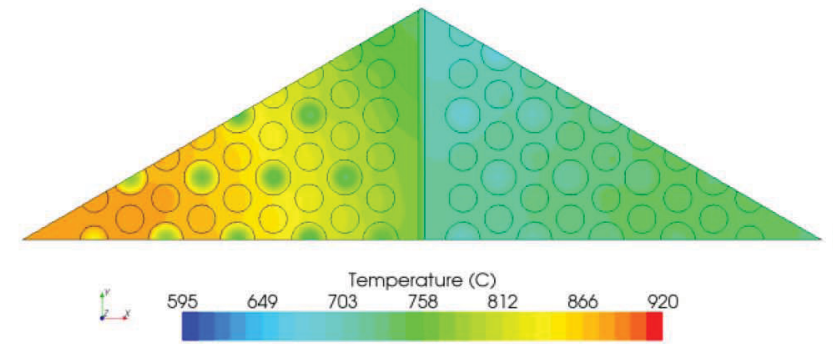

(a)

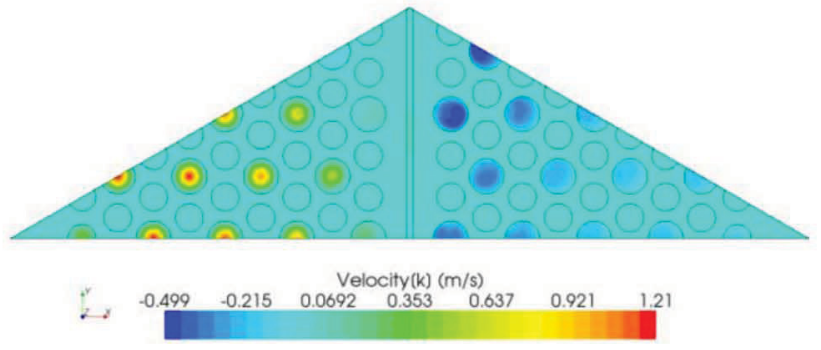

(b)

Figure 5. Contours of (a) temperature at the hot plane and (b) velocity at top of the core after 100 seconds.

For these simulations, it is assumed that the coolant circulators are immediately cut off. In reality, it will take some time (presumably some few minutes) for the circulators to stop completely. Figure 6 shows the velocities in the initial 100 seconds of the transient in three channels on each side of the bypass gap. It can be seen, that once the circulators are shut down, the transient becomes "quasi-steady" fairly quickly, and then doesn't change by much after the initial rapid transient. Figure 7 plots streamlines at 100 seconds into the transient in the upper plenum, which is a truncated $0.35 \mathrm{~m} \mathrm{high}$. It can be seen that fairly strong circulation paths that span the two sectors are established. There were three configurations of geometry considered in the study of Ref. [3]: a single one-twelfth sector, a 1/6 sector and the present 2 $\times 1 / 12$ sector geometry. Of the three configurations, the $2 \times 1 / 12$ sector geometry produced by far the most vigorous natural circulation.

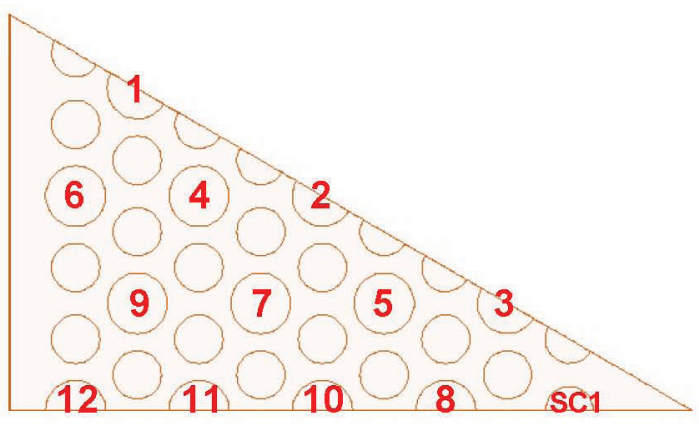

(a)

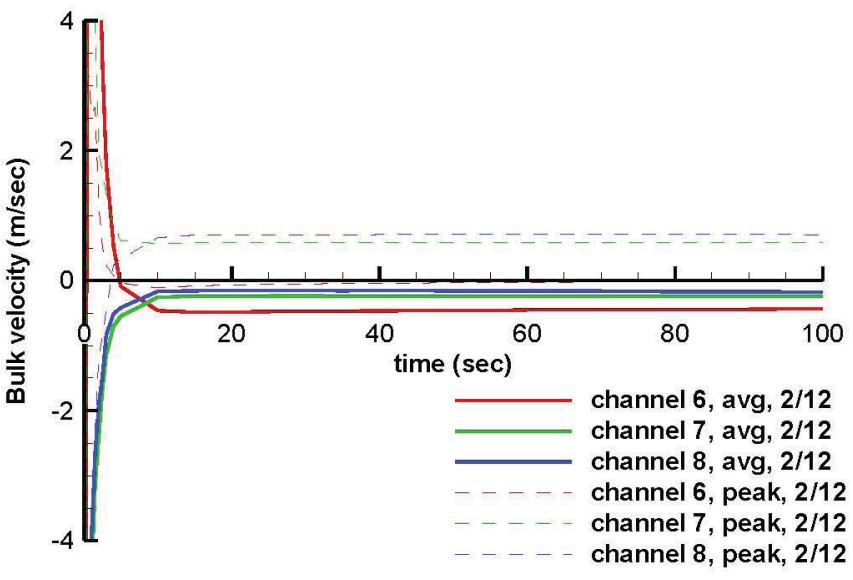

(b)

Figure 6. (a) Coolant channel reference numbers and (b) transient bulk velocities in three of the channels for the hot and cool sectors. 


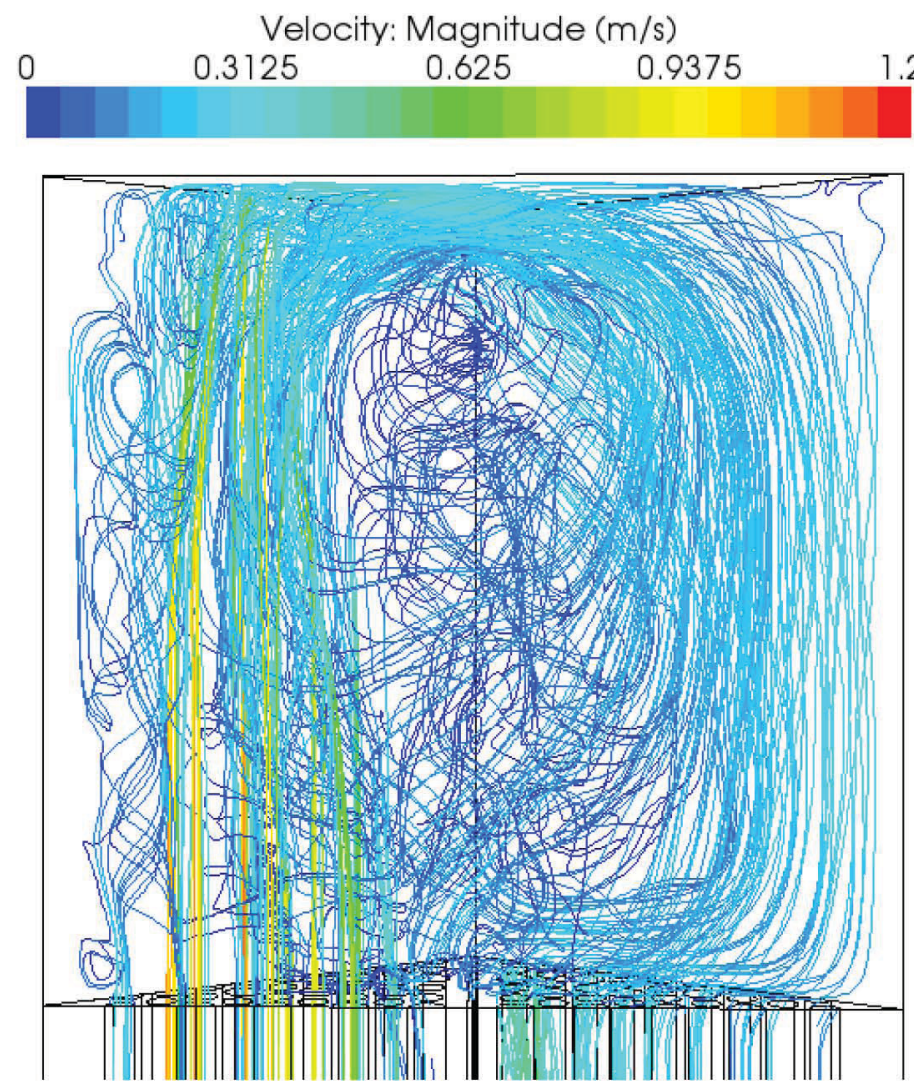

Figure 7. Streamline plot of the upper plenum at $100 \mathrm{sec}$.

Again, the flow and heat transfer for the case discussed above started with a steady-state normal operating condition at full power. The power is then cut to $10 \%$ of normal operation and the coolant circulators are immediately stopped at the beginning of the LOFA accident transient. Natural circulation is then allowed to become established, which develops into a "quasi-steady" condition, that is, with very slowly varying conditions. The heat transfer in the core can therefore be described as starting from a fully forced convection situation characterized by turbulent flow. At the moment the circulators are lost, there begins a fast transient period wherein the flow transitions from forced to unforced over a short period of presumably a few minutes. During this time the heat transfer is transitioning from fully forced convection to mixed convection and eventually to free convection as the circulators fully stop and buoyancy takes over as the main driving force. During the time when there is forced flow, the characteristic velocity is given by the bulk velocity of the forced flow. However, when there is no forced flow, the characteristic velocity changes to one based on fluid parameters, according to White [2]; that is $V_{o} \sim \mu_{o}\left(\rho_{o} L\right)$, where $L$ $=D$, and, hence, the Reynolds number is identically equal to unity, even though there is still a bulk velocity caused by the buoyant forces during free convection.

The dimensionless parameters discussed above for these transitioning conditions have been computed from the above $2 \times 1 / 12$ sector case and are given below in Table 1. Reference properties are computed from conditions that are present in the lower plenum at the time of the initiation of the LOFA. Note that the temperature in the lower plenum is not constant. Hence a representative value is used. The reference temperature and pressure used are $1300 \mathrm{~K}$ and 6.4 MPa (for helium). For reference, the Reynolds number 
using the resulting bulk velocity is also given. It is apparent that the flow is still laminar. See Patel and Head [6]. Swapnalee and Vijayan [7] develop a natural circulation loop equation that relates the Reynolds number (based on the actual mass flow rate or bulk velocity) to Grashof number: $\operatorname{Re}=$ $\mathrm{C}\left[\mathrm{Gr} / \mathrm{N}_{\mathrm{G}}\right]^{\mathrm{r}}$, where $\mathrm{N}_{\mathrm{G}}$ represents the friction in the loop, which may change nature depending on the location in the loop. It is apparent from their equation that Reynolds and Grashof numbers are the defining parameters for natural circulation, though they employ one-dimensional equations, unlike the CFD calculations discussed above.

Table 1. Flow and heat transfer parameters before and during a LOFA.

\begin{tabular}{|c|c|c|c|c|}
\hline Parameter & $\operatorname{Re}$ & $\mathrm{Gr}$ & $\mathrm{Gr} / \mathrm{Re}^{2}$ & $\operatorname{Pr}$ \\
\hline Normal operation & $25,000-55,000$ & 65,300 & $0.0001-0.00002$ & $\sim 0.65$ \\
\hline $\begin{array}{c}\text { Power down } \\
\text { transient (rapid) }\end{array}$ & $55,000-1$ & $65,300-45,200$ & $0.00002-45,200$ & $\sim 0.65$ \\
\hline $\begin{array}{l}\text { Free convection } \\
\text { transient (slow) }\end{array}$ & 1 & 45,200 & 45,200 & $\sim 0.65$ \\
\hline $\begin{array}{l}\text { Free convection } \\
\text { transient with } V_{o} \\
\text { based on bulk } \\
\text { velocity }\end{array}$ & $\begin{array}{c}\sim 1100 \\
\text { (laminar flow) }\end{array}$ & same & N/A & same \\
\hline
\end{tabular}

\section{References}

[1] Currie, I. G., 1974, Fundamental Mechanics of Fluids, McGraw-Hill, Inc., New York.

[2] White, F. M., 1991, Viscous Fluid Flow, $2^{\text {nd }}$ ed., McGraw-Hill, Inc., New York.

[3] Tung, Y-H., and R. W. Johnson, 2011, "CFD Calculations of Natural Circulation in a High Temperature Gas Reactor Following Pressurized Circulator Shutdown," paper IMECE2011-64259, ASME 2011 IMECE, Denver, Colorado, Nov. 11-17, 2011.

[4] General Atomics, 1977, Safety Analysis Report use of H451 Graphite in Fort St. Vrain Fuel Elements, GLP-5588.

[5] MacDonald, P., J. Sterbentz, R. Sant, P. Bayless, R. Schultz, H. Gougar, R. Moore, A. Ougouag, W. Terry, 2003, NGNP Preliminary Point Design Results of the Initial Neutronics and Thermal-Hydraulic Assessments, INEEL/EXT-03-00870.

[6] Patel, V. C. and Head, M. R., 1969, Some observations on skin friction and velocity profiles in fully developed pipe and channel flows, J. Fluid Mech., 38(1), 181-201.

[7] Swapnalee, B. T., and Vijayan, P. K., 2011, A generalized flow equation for single phase natural circulation loops obeying multiple friction laws, Int. J. Heat Mass Trans., 54, 2618-2629. 